\title{
Phase diagram of alpha matter with Skyrme-like scalar interaction
}

\author{
L. M. Satarov ${ }^{1}$ R. V. Poberezhnyuk, ${ }^{1,2}$ I. N. Mishustin, ${ }^{1,3}$ and H. Stoecker ${ }^{1,4,5}$ \\ ${ }^{1}$ Frankfurt Institute for Advanced Studies, \\ D-60438 Frankfurt am Main, Germany \\ ${ }^{2}$ Bogolyubov Institute for Theoretical Physics, 03680 Kiev, Ukraine \\ ${ }^{3}$ National Research Center "Kurchatov Institute" 123182 Moscow, Russia \\ ${ }^{4}$ Institut für Theoretische Physik, Goethe Universität Frankfurt, \\ D-60438 Frankfurt am Main, Germany \\ ${ }^{5}$ GSI Helmholtzzentrum für Schwerionenforschung GmbH, D-64291 Darmstadt, Germany

\begin{abstract}
The equation of state and phase diagram of strongly interacting matter composed of $\alpha$ particles are studied in the mean-field approximation. The particle interactions are included via a Skyrme-like mean field, containing both attractive and repulsive terms. The model parameters are found by fitting the values of binding energy and baryon density in the ground state of $\alpha$ matter, obtained from microscopic calculations by Clark and Wang [Ann. Phys. (NY) 40, 127 (1966)]. Thermodynamic quantities of $\alpha$ matter are calculated in the broad domains of temperature and baryon density, which can be reached in heavy-ion collisions at intermediate energies. The model predicts both first-order liquid-gas phase transition and Bose-Einstein condensation of $\alpha$ particles. We present the profiles of scaled variance, sound velocity and isochoric heat capacity along the isentropic trajectories of $\alpha$ matter. Strong density fluctuations are predicted in the vicinity of the critical point at temperature $T_{c} \approx 14 \mathrm{MeV}$ and density $n_{c} \approx 0.012 \mathrm{fm}^{-3}$.
\end{abstract}




\section{INTRODUCTION}

It is well known from experimental observations [1-3] and theoretical studies [4-7] that symmetric nuclear matter at subsaturation densities has a tendency for clusterization. And $\alpha$ particles are the most abundant clusters, as observed in many experiments $[8,9]$. The fact that $\alpha$ particles are bosons makes $\alpha$ matter even more interesting to study, because of the possibility of Bose-Einstein condensation (BEC).

A special role of $\alpha$ particles is well established in nuclear physics. The examples include: $2 \alpha$ structure of ${ }^{8} \mathrm{Be}$ which decays into two $\alpha$ 's in about $10^{-16} s$ and the famous $3 \alpha$ Hoyle state in ${ }^{12} \mathrm{C}$ with width of only $8.5 \mathrm{eV}$. Recently, $\alpha$-clustered excited states, analogous to the Hoyle state, have been identified in ${ }^{16} \mathrm{O}$ and ${ }^{24} \mathrm{Mg}$ [10, 11]. In Ref. [12] an evidence for $7 \alpha$ resonance has been reported. All these states can be interpreted as Bose condensates of $\alpha$ particles in finite systems $[13,14]$.

Early theoretical studies of clusterized nuclear matter applied variational calculations with phenomenological two-body potentials. For example, in Ref. [15] Clark and Wang calculated energy per baryon for pure $\alpha$ matter by using several potentials of $\alpha \alpha$ interaction. They have found that such matter has a ground state (GS) with the binding energy, only several $\mathrm{MeV}$ (per baryon) smaller than the iso-symmetric nuclear matter. It was demonstrated [16] that this binding energy is sensitive to the short-range behavior of the potential.

One should also mention the virial [17] and quasiparticle [18, 19] models. In particular, in Ref. [17], the authors express the pressure and partial densities of $\alpha-N$ matter via integrals over observed phase shifts of $N N, \alpha N$ and $\alpha \alpha$ scattering. Although such an approach is 'model-independent', it can be applied only for dilute matter.

Significant progress in modern computing has stimulated 'ab-initio' microscopic calculations of nuclei, which use realistic $2 N$ and $3 N$ forces to describe cluster degrees of freedom (for a recent review, see Ref. [20]). Such studies include, e.g., the antisymmetrized [21] and fermionic [22] molecular dynamics, the quantum Monte Carlo approach [23] and the nuclear lattice effective theory [24, 25]. In particular, it has been shown in Ref. [25] that increasing the depth of the nucleon-nucleon potential at short distances (without significantly changing the $N N$ and $3 N$ data) leads to formation of a clusterized phase in GS of $\alpha$-congugate nuclei $(N=Z \geqslant 4)$. The above-mentioned microscopic methods are computationally demanding and currently may deal only with light- and medium-size nuclei. On the other hand, they 
consistently take into account short-range correlations of nucleons and in-medium modification of clusters.

Recently another microscopic method for studying clusterization phomena in nuclei has been proposed [26-28], namely the nuclear density functional theory (DFT). In fact, it is an extension of the mean-field approach. Within the DFT, clusters are associated with spatially localized fluctuations of nucleon density. As demonstrated in Ref. [27], main features of $\alpha$-conjugate nuclei can be well described within this approach.

Several versions of the relativistic mean-field (RMF) model have been proposed to describe clusterized nuclear matter (see, e.g., Refs. [29-31]). Due to neglect of NN correlations, clusters are introduced in this model as separate degrees of freedom. This leads to appearance of additional model parameters characterizing interactions of clusters and nucleons. In Refs. [30, 31], the authors additionally introduce shifts of cluster masses to implement disappearance of clusters at high baryon densities.

The possibility of the Bose-Einstein cluster condensation in stellar matter at nonzero temperatures has been studied within the quantum-statistical model in Ref. [32]. Attempts to implement quantum statistics for nucleons and nuclear clusters have been also made in the quasiclassical molecular dynamical model $[6,33,34]$. This was achieved by adding the phenomenological Pauli potential which becomes strongly repulsive for nucleon pairs with close phase-space coordinates. In Ref. [34] a resonable desciption of light cluster properties has been obtained within this approach.

In our previuos works [35-37] several mean-field models with Skyrme-like vector potentials have been developed to describe simultaneously the liquid-gas phase transition (LGPT) and BEC in $\alpha$ and $\alpha-N$ matter. In the present paper, we propose another approach where $\alpha$-clustered nuclear matter is described by a scalar field $\phi$ with an effective Lagrangian containing the attractive $\left(\phi^{4}\right)$ and repulsive $\left(\phi^{6}\right)$ terms. Analogous scalar potential was first introduced by Boguta et al. in the RMF model of nuclear matter [38, 39]. In Ref. [40], a similar model was used for bosonic particles without any conserved charge, i.e., with zero chemical potential (e.g., pions). In the present paper we extend this approach for $\alpha$ particles which carry the conserved baryon charge.

The paper is organized as follows. In Section II we formulate the model. In Sec. III we analyze the phase diagram of $\alpha$ matter which contains both LGPT and BEC. We also discuss isentropic and isothermal trajectories of $\alpha$ matter in different thermodynamic variables. 
In Sec. IV A we present a fluctuation observable in terms of the scaled variance $\omega$, and show that it has a strong peak at the critical point of liquid-gas phase transition. In Sec. IV B and IV $\mathrm{C}$ we calculate isentropic profiles of the sound velocity and the isochoric heat capacity. It is shown that both these quantities exhibit jumps at the mixed phase boundary.

\section{THE MODEL}

\section{A. Motivation}

In Refs. [36, 37], we have studied nuclear systems composed of $\alpha$ particles and nucleons. Two possibilities regarding the interaction between $\alpha$ particles and nucleons have been considered. In the first scenario [36], pure $\alpha$ matter and pure nucleonic matter have their own GSs separated by a potential barrier. In this case the resulting phase diagram has two LGPTs: one (metastable) in the domain with large $\alpha$ abundances and another one (stable) in the domain dominated by nucleons. In Ref. [37] we have analyzed the sensitivity of nuclear matter phase diagram to the strength of $\alpha N$ interaction. Within the mean-field approximation (MFA), a threshold-like behavior of $\alpha$ clustering in the cold nuclear matter has been predicted. This effect is similar to the 'quantum phase transition' discovered in Ref. [25]. We show that the energy barrier separating the clusterized- and non-clusterized phases disappears at some critical strength of the $\alpha N$ attraction. In this case, there is only one common GS with coexisting nucleons and $\alpha$ 's in chemical equilibrium, but the admixture of $\alpha$ particles is small at moderate temperatures and densities.

Having in mind that experimental data on heavy-ion collisions at intermediate energies $[10,12]$ show enhanced yields of $\alpha$ particles and $\alpha$-conjugate nuclei, we are tempted to conclude that the first scenario is more realistic. In this case, there is a chance that the excited matter produced in such collisions will expand along a metastable branch of the phase diagram where $\alpha$ particles are more abundant and even may form a condensate. In the present paper, we study this scenario within a Skyrme-type mean-field model for pure $\alpha$ matter, i.e., ignoring the admixture of nucleons. 


\section{B. Bosonic matter in the mean-field approximation}

Following Ref. [40] we describe the system of scalar bosons with zero spin $^{1}$ by a scalar field operator $\phi(x)$ with the Lagrangian $(\hbar=c=1)$

$$
\mathcal{L}=\frac{1}{2}\left(\partial_{\mu} \phi \partial^{\mu} \phi-m^{2} \phi^{2}\right)+\mathcal{L}_{\text {int }}\left(\phi^{2}\right)
$$

Here $m$ is the boson mass in the vacuum and $\mathcal{L}_{\text {int }}$ contain particles' interactions. In the MFA one expands $\mathcal{L}_{\text {int }}$ in the lowest order in $\phi^{2}-\sigma$, where

$$
\sigma=\left\langle\phi^{2}\right\rangle
$$

is the scalar density (c-number) and angular brackets denote the quantum-statistical averaging in the grand-canonical ensemble (see below).

One gets the following result

$$
\mathcal{L} \approx \mathcal{L}_{\mathrm{MFA}}=\frac{1}{2}\left[\partial_{\mu} \phi \partial^{\mu} \phi-M^{2}(\sigma) \phi^{2}\right]+p_{\mathrm{ex}}(\sigma)
$$

where the quantity

$$
M^{2}(\sigma)=m^{2}-2 \mathcal{L}_{\text {int }}^{\prime}(\sigma)
$$

is the effective mass squared, and

$$
p_{\text {ex }}(\sigma)=\mathcal{L}_{\text {int }}(\sigma)-\sigma \mathcal{L}_{\text {int }}^{\prime}(\sigma)
$$

is the so-called excess pressure [40] (hereinafter primes denote derivatives over $\sigma$ ). The terms containing $\mathcal{L}_{\text {int }}$ in these two equations describe deviations from the ideal gas. Later on we apply the Skyrme-like parametrization for $\mathcal{L}_{\text {int }}(\sigma)$ containing both the attractive and repulsive parts. From Eqs. (4), (5) one gets the differential relation between the effective mass and excess pressure

$$
p_{\mathrm{ex}}^{\prime}(\sigma)=\sigma M M^{\prime}(\sigma)
$$

which guaranties the thermodynamic consistency of the present model (see below). In fact, the appearance of the effective mass makes the main difference between the present approach and our previous studies of $\alpha[35]$ and $\alpha-N[36,37]$ matter.

\footnotetext{
${ }^{1}$ All numerical calculations below are carried out for the specific case of $\alpha$ particles.
} 
Using a plane-wave decomposition of $\phi$ in terms of creation $\left(a_{\boldsymbol{k}}^{+}\right)$and annihilation $\left(a_{\boldsymbol{k}}\right)$ operators $^{2}$

$$
\phi(x)=\frac{g}{(2 \pi)^{3}} \int \frac{d^{3} k}{\sqrt{2 E_{k}}}\left(a_{\boldsymbol{k}} e^{-i k x}+a_{\boldsymbol{k}}^{+} e^{i k x}\right),
$$

where $k^{0}=E_{\boldsymbol{k}}=\sqrt{M^{2}+\boldsymbol{k}^{2}}$ and $g$ is the statistical weight of a boson particle, one gets [40] the following equations for the particle number and Hamiltonian density operators in the MFA

$$
\frac{N}{V}=\frac{g}{(2 \pi)^{3}} \int d^{3} k a_{\boldsymbol{k}}^{+} a_{\boldsymbol{k}}, \quad \frac{H}{V}=\frac{g}{(2 \pi)^{3}} \int d^{3} k E_{\boldsymbol{k}} a_{\boldsymbol{k}}^{+} a_{\boldsymbol{k}}-p_{\mathrm{ex}} .
$$

In the grand canonical ensemble one can find the particle momentum distribution by averaging $a_{\boldsymbol{k}}^{+} a_{\boldsymbol{k}}$ over the statistical operator $\rho \propto \exp [(\mu N-H) / T]$ at given chemical potential $\mu$ and temperature $T$. Within the MFA one has

$$
n_{\boldsymbol{k}}=\left\langle a_{\boldsymbol{k}}^{+} a_{\boldsymbol{k}}\right\rangle=\left[\exp \left(\frac{E_{\boldsymbol{k}}-\mu}{T}\right)-1\right]^{-1} .
$$

One can see that this distribution coincides with the ideal-gas (Bose-Einstein) distribution of quasiparticles with mass $M$. The latter is in general not equal to $m$ and should be found self-consistently at given $T$ and $\mu$. According to Eq. (9) possible values of $\mu$ are bound from above, namely $\mu \leqslant M$. In the particular case $\mu=M$ the distribution $n_{\boldsymbol{k}}$ is singular at $\boldsymbol{k}=0$. Similarly to the ideal-gas case, this implies the appearance of BEC with macroscopic number of zero momentum particles. Therefore, in our model the condensate appears at $\mu=M(\sigma)$. We will see that this takes place either at low enough temperatures or at sufficiently large densities.

Using Eqs. (2), (7), (9) one can calculate the scalar density $\sigma$ :

$$
\sigma=\sigma_{\mathrm{th}}[T, \mu, M(\sigma)]
$$

where

$$
\sigma_{\mathrm{th}}(T, \mu, M)=\frac{g}{(2 \pi)^{3}} \int d^{3} k \frac{n_{\boldsymbol{k}}}{E_{\boldsymbol{k}}}=\frac{g}{2 \pi^{2}} \int_{M}^{\infty} \frac{d E \sqrt{E^{2}-M^{2}}}{\exp \left(\frac{E-\mu}{T}\right)-1} .
$$

In fact, Eq. (10) plays a role of self-consistent gap equation which determines $\sigma$ and $M$ as functions of $T, \mu$.

\footnotetext{
${ }^{2}$ For states with BEC one should add [40] an additional condensate component $\phi_{c}$ to the right-hand side (r.h.s.) of this equation.
} 
The pressure $p$ is determined by spatial components of the energy-momentum tensor $T_{\alpha \alpha}$ which in turn can be calculated from the Lagrangian. Within the MFA one has

$$
p=\frac{1}{3}\left\langle T_{x x}+T_{y y}+T_{z z}\right\rangle=\frac{1}{6}\left\langle(\nabla \phi)^{2}\right\rangle+p_{\text {ex }}(\sigma) .
$$

Using Eqs. (7), (9) one can easily calculate the kinetic term in the second equality (below we denote it by $\left.p_{\text {th }}\right)$. Finally one gets the equation

$$
p=p_{\text {th }}(T, \mu, M)+p_{\text {ex }}(\sigma),
$$

where

$$
p_{\text {th }}=\frac{g}{(2 \pi)^{3}} \int d^{3} k \frac{\boldsymbol{k}^{2}}{3 E_{\boldsymbol{k}}} n_{\boldsymbol{k}}=\frac{g}{6 \pi^{2}} \int_{M}^{\infty} \frac{d E\left(E^{2}-M^{2}\right)^{3 / 2}}{\exp \left(\frac{E-\mu}{T}\right)-1} .
$$

One can also obtain explicit expressions for the number density of $\alpha$ particles $n=\langle N\rangle / V$ and the internal energy density $\varepsilon=\langle H\rangle / V$ (note that the baryon density $n_{B}=4 n$ ). Using Eqs. (8), (9) one has

$$
n=n_{\mathrm{th}}(T, \mu, M)=\frac{g}{(2 \pi)^{3}} \int d^{3} k n_{\boldsymbol{k}}
$$

and

$$
\varepsilon=\varepsilon_{\mathrm{th}}(T, \mu, M)-p_{\mathrm{ex}}(\sigma), \quad \varepsilon_{\mathrm{th}}=\frac{g}{(2 \pi)^{3}} \int d^{3} k E_{\boldsymbol{k}} n_{\boldsymbol{k}} .
$$

In the case of $\alpha$ matter, the temperatures of interest are lower or of the order of $\alpha$-particle binding energy $(\approx 28 \mathrm{MeV})$. To a good accuracy, one can apply the non-relativistic approximation (NRA) by taking the lowest-order terms in $T / M$ in Eqs. (11), (14)-(16). This leads to approximate relations

$$
\begin{aligned}
\sigma_{\mathrm{th}} & \approx \frac{n_{\mathrm{th}}}{M} \approx \frac{g}{M \lambda_{T}^{3}} g_{3 / 2}(z), \\
p_{\mathrm{th}} & \approx \frac{2}{3}\left(\varepsilon_{\mathrm{th}}-M n_{\mathrm{th}}\right) \approx \frac{g T}{\lambda_{T}^{3}} g_{5 / 2}(z),
\end{aligned}
$$

where $z \leqslant 1$ is a nonrelativistic fugacity, and $\lambda_{T}=\lambda_{T}(T, M)$ is the thermal wave length:

$$
z \equiv \exp \left(\frac{\mu-M}{T}\right), \quad \lambda_{T}=\sqrt{\frac{2 \pi}{M T}} .
$$

The dimensionless function (polylogarithm) $g_{\beta}(z)$ is defined as

$$
g_{\beta}(z) \equiv \frac{1}{\Gamma(\beta)} \int_{0}^{\infty} d x \frac{x^{\beta-1}}{z^{-1} e^{x}-1}=\sum_{k=1}^{\infty} z^{k} k^{-\beta},
$$


where $\Gamma(\beta)$ is the gamma function.

The following properties of the polylogarithm will be used below:

$$
z g_{\beta}^{\prime}(z)=g_{\beta-1}(z), \quad g_{\beta}(z)= \begin{cases}z, & z \ll 1, \\ \xi(\beta), & z \rightarrow 1,\end{cases}
$$

where $\xi(\beta)=\sum_{k=1}^{\infty} k^{-\beta}$ is the Riemann function. The function $g_{\beta}(z)$ diverges at $z \rightarrow 1$ if $\beta \leqslant 1$. The classical (Boltzmann) limit corresponds to small $z$. According to Eqs. (17) and (21), in this case $z \approx n \lambda_{T}^{3} / g \ll 1$ and $p_{\text {th }} \approx n T$.

On the other hand, for states where the degeneracy parameter $n \lambda_{T}^{3} \gtrsim 1$, the effects of quantum statistics are important. As discussed above, the BEC starts when $\mu \rightarrow M$, i.e., at $z \rightarrow 1$. From Eqs. (17), (19) and (21) one can see that this happens at $T<T_{\mathrm{BEC}}$ where

$$
T_{\mathrm{BEC}} \approx \frac{2 \pi}{\mu}\left(\frac{n}{g \xi(3 / 2)}\right)^{2 / 3}
$$

is the threshold temperature of BEC. The chemical potential in the r.h.s. of this equation is determined from the relation $\mu=M(\sigma)$. At not too high densities one can take $\mu \approx m$ with a good accuracy. The resulting value of $T_{\mathrm{BEC}}$ coincides with that for the ideal Bose gas. Therefore, for nonrelativistic bosons (like $\alpha$-particles) the BEC onset line in the $(n, T)$ plane is the same as in the ideal gas [35, 41].

At $T<T_{\mathrm{BEC}}$ additional condensate terms should be added to the r.h.s. of Eqs. (10), (15) and $(16)^{3}$ :

$$
\sigma(\mu)=\sigma_{c}+\sigma_{\mathrm{th}}(T, \mu, \mu), \quad n=n_{c}+n_{\mathrm{th}}(T, \mu, \mu), \quad \varepsilon=\varepsilon_{c}+\varepsilon_{\mathrm{th}}(T, \mu, \mu)-p_{\mathrm{ex}}(\sigma),
$$

where $n_{c}=\mu \sigma_{c}, \varepsilon_{c}=\mu n_{c}$ and $\sigma(\mu)$ is determined by solving the equation $\mu=M(\sigma)$. Note that $(\mu, T)$ dependence of the condensate terms is fully determined by the first equality in $(23)$.

Let us consider now the entropy of a bosonic system $S$. The entropy density $s=S / V$ can be calculated by using the general relation $s=(\varepsilon+p-\mu n) / T$. One can see that the excessand (possible) condensate terms in $\varepsilon, p$ and $n$ are cancelled and we arrive at the equation:

$$
s=\frac{\varepsilon_{\mathrm{th}}+p_{\mathrm{th}}-\mu n_{\mathrm{th}}}{T} .
$$

\footnotetext{
${ }^{3}$ By presence of BEC the pressure is modified only indirectly, via the condensate scalar density $\sigma_{c}$ in the term $p_{\text {ex }}(\sigma)$.
} 
Formally, we get the same expression as for the ideal Bose gas [40]. However, the interaction effects enter via the effective mass $M$. Substituting $\mu=M+T \ln z$ and using Eqs. (17), (18) one obtains the following expression for the entropy per particle $\widetilde{s}=s / n$

$$
\widetilde{s}=\frac{S}{N} \approx \begin{cases}\frac{5}{2} \frac{g_{5 / 2}(z)}{g_{3 / 2}(z)}-\ln z, & T>T_{\mathrm{BEC}} \\ \frac{5}{2} \frac{\xi(5 / 2)}{n \lambda_{T}^{3}(T, \mu)}, & T<T_{\mathrm{BEC}} .\end{cases}
$$

Again, we arrive at the relations for the ideal Bose gas [42], but with the modified (statedependent) particle mass. These results show that the specific entropy $\widetilde{s}$ is constant at the $\operatorname{BEC}$ boundary $(z=1)$ :

$$
\widetilde{s} \approx \frac{5}{2} \frac{\xi(5 / 2)}{\xi(3 / 2)} \approx 1.284 \quad\left(T=T_{\mathrm{BEC}}\right)
$$

This means that this boundary is an isentrope and the bosonic matter can not cross it during the isentropic evolution. For example, it is not possible to reach the BEC region by an adiabatic expansion of $\alpha$ matter from non-condensed initial states ${ }^{4}$. Note that the threshold value (26) does not depend on the particle mass and the interaction parameters.

Within the MFA one obtains the standard thermodynamic relations [43]

$$
\begin{aligned}
& d p=s d T+n d \mu \\
& d \varepsilon=T d s+\mu d n
\end{aligned}
$$

To prove (27), we directly calculate the derivatives entering the pressure differential $d p=d T \partial p / \partial T+d \mu \partial p / \partial \mu+d M \partial p / \partial M$. Using Eqs. (13)-(16), (24) one can check that $\partial p / \partial T=\partial p_{\text {th }} / \partial T=s, \partial p / \partial \mu=\partial p_{\text {th }} / \partial \mu=n_{\text {th }}$ and $[40]$

$$
\frac{\partial p}{\partial M}=\frac{\partial\left(p_{\mathrm{th}}+p_{\mathrm{ex}}\right)}{\partial M}=-M \sigma_{\mathrm{th}}+M \sigma= \begin{cases}0, & T>T_{\mathrm{BEC}} \\ \mu \sigma_{c}, & T<T_{\mathrm{BEC}}\end{cases}
$$

where we have used Eqs. (6) and (23) (in the second and third equalities, respectively). Finally we arrive at Eq. (27). Note that in the BEC region we substitute $M=\mu$ and $\mu \sigma_{c}=n-n_{\text {th }}$. Equation (28) can be obtained from (27) by differentiating the relation $\varepsilon=T s+\mu n-p$.

\footnotetext{
${ }^{4}$ However, this is possible if the bosonic matter contains also the LGPT (see Sec. III).
} 


\section{Skyrme-like parametrization of particle interactions in $\alpha$ matter}

The above results are obtained in the MFA, and they do not depend on a specific form of the interaction Lagrangian. The following calculations are carried out with the Skyrme-like $\left(\phi^{4}-\phi^{6}\right)$ parametrization,

$$
\mathcal{L}_{\text {int }}(\sigma)=\frac{a}{4} \sigma^{2}-\frac{b}{6} \sigma^{3},
$$

where $a, b$ are positive constants. The first and the second terms in (30) describe, respectively, the attractive and the repulsive interactions between the scalar bosons. It will be shown below that the presence of attraction leads to the appearance of the first-order (liquidgas) phase transition of bosonic matter. On the other hand, the repulsive term stabilizes this matter at high densities. Substituting (30) into Eqs. (4) and (5) gives the explicit relations

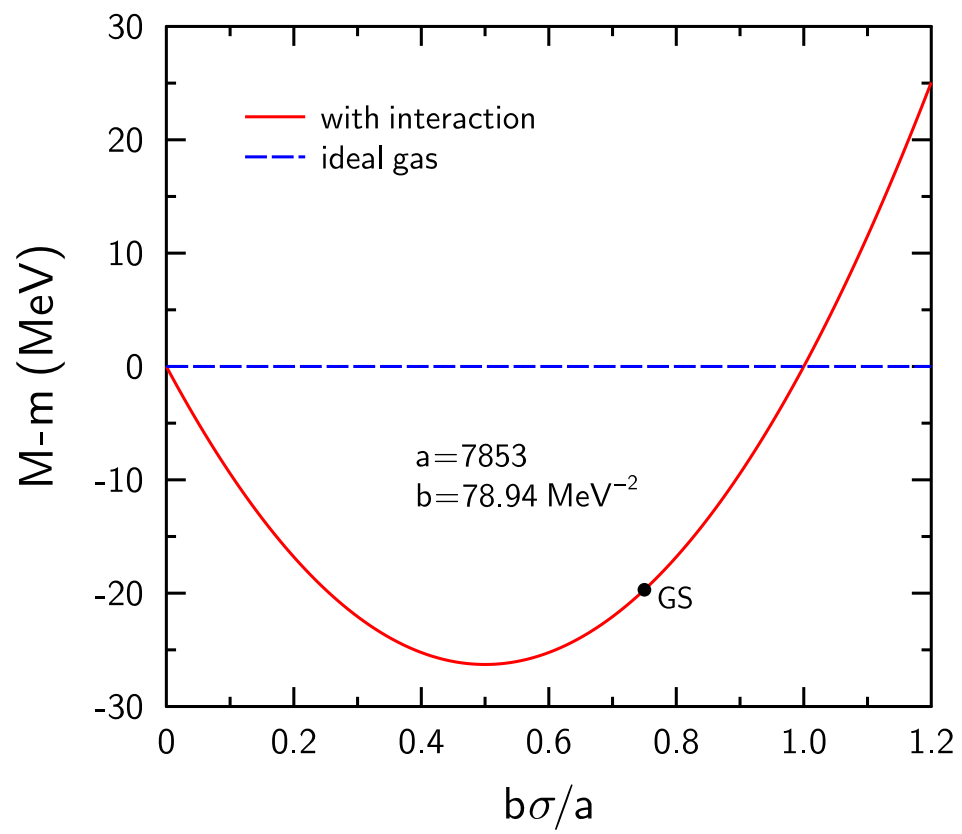

FIG. 1. Effective mass of particles in $\alpha$ matter as the function of scalar density $\sigma$. Full dot shows position of GS .

for the effective mass and excess pressure ${ }^{5}$

$$
\begin{aligned}
& M(\sigma)=\sqrt{m^{2}-a \sigma+b \sigma^{2}}, \\
& p_{\text {ex }}(\sigma)=-\frac{a}{4} \sigma^{2}+\frac{b}{3} \sigma^{3} .
\end{aligned}
$$

${ }^{5}$ A similar quasiparticle model for scalar bosons with repulsive interaction was constructed in Ref. [44]. 
Note that $p_{\text {ex }}$ vanishes at $\sigma=\frac{3 a}{4 b}$ and the minimum value of the effective mass $M_{\min }=\sqrt{m^{2}-\frac{a^{2}}{4 b}}$ is reached at $\sigma=\frac{a}{2 b}$. A typical plot of $M(\sigma)$ for the case of $\alpha$ matter $(m=3727.3 \mathrm{MeV})$ is shown in Fig. 1. It is seen that at not too high densities the deviation of $M$ from its vacuum value at $\sigma \rightarrow 0$ is relatively small.

Similarly to Ref. [36] we fix the interaction parameters $a, b$ by fitting known properties of cold $\alpha$ matter. According to microscopic calculations of Ref. [15] this matter has the following values of the binding energy per $\alpha$ particle ${ }^{6} W_{0}$ and the equilibrium density $n_{0}$ :

$$
W_{0}=m-\left(\frac{\varepsilon}{n}\right)_{\min }=19.7 \mathrm{MeV}, \quad n_{0}=0.036 \mathrm{fm}^{-3}
$$

The bosonic matter at $T=0$ consists of condensed quasiparticles with $\mu=M(\sigma)$, $n=n_{c}=\mu \sigma$, and $p=p_{\text {ex }}(\sigma)$. For the GS one has $d\left(\frac{\varepsilon}{n}\right) / d n=p / n^{2}=0$ and we obtain the following equations connecting equilibrium parameters $n_{0}, W_{0}$ and $\sigma_{0}$ :

$$
p_{\mathrm{ex}}\left(\sigma_{0}\right)=0, \quad m-W_{0}=M\left(\sigma_{0}\right)=\frac{n_{0}}{\sigma_{0}}
$$

As already mentioned, the first equation holds at $\sigma_{0}=\frac{3 a}{4 b}$. Solving two remaining equalities, one obtains the following values of parameters $a, b$ :

$$
a \approx 7853, \quad b \approx 78.94 \mathrm{MeV}^{-2}
$$

Note, that the position of GS is indicated by dot in Fig. 1 . The GS value $\sigma_{0} \approx 75 \mathrm{MeV}^{2}$ is significantly lower than the close packing density $\sigma_{p} \sim 200 \mathrm{MeV}^{2}$ (it corresponds to the vector density $\left.n_{p} \approx m \sigma_{p} \sim 0.1 \mathrm{fm}^{-3}[36]\right)$.

It is instructive to calculate the incompressibility modulus:

$$
K_{0}=9 \frac{d p}{d n} \approx \frac{9 p_{\mathrm{ex}}^{\prime}\left(\sigma_{0}\right)}{m}=\frac{27}{16} \frac{a^{2}}{b m} \approx 354 \mathrm{MeV}
$$

This agrees with the value obtained in Ref. [36] for a pure $\alpha$ matter with a mean-field vector potential $U(n)$. The latter is defined as the shift of chemical potential with respect to the ideal gas. One can show that within the NRA such an approach gives results which are similar to the present model with scalar interaction. Indeed, at $T=0$ the chemical potential

\footnotetext{
${ }^{6}$ The corresponding binding energy per nucleon, $W=m_{N}+\left(W_{0}-m\right) / 4 \approx 12 \mathrm{MeV}$ is slightly less than the binding energy of isosymmetric nuclear matter (about $16 \mathrm{MeV} /$ nucleon).
} 
in the scalar theory equals $M(\sigma)$ where $\sigma \approx n / m$. Decomposing the r.h.s. of Eq. (31) in powers of $\sigma$ one obtains the Skyrme-like expression ${ }^{7}$ for the equivalent vector potential:

$$
U(n) \approx M(\sigma)-m \approx-A n+B n^{2}, \text { where } A=\frac{a}{2 m^{2}}, B=\frac{b}{2 m^{3}} .
$$

Substituting the values of $a$ and $b$ from (35), we get the values of $A, B$ close to those obtained in Ref. [36]. However, one can show that the approach with vector potential (37) leads to superluminal sound velocities $c_{s}$ at sufficiently large $n$. On the other hand, the present model does not violate the relativistic causality condition $c_{s}<1$ for all equilibrium states.

\section{PHASE DIAGRAM OF $\alpha$ MATTER}

By using the model with vector mean-field interaction we have calculated in Refs. [35, 36] the phase diagram of the bosonic matter. It was demonstrated that if this interaction includes both attractive and repulsive term, the resulting phase diagram contains both regions of LGPT and BEC. Below we show that a similar phase diagram takes place in the present model with scalar interaction.

\section{A. The BEC boundary}

As explained above, the BEC boundary in the $(\mu, T)$ plane, $T=T_{\mathrm{BEC}}(\mu)$, is determined by simultaneous solving the equations $M(\sigma)=\mu, \sigma=\sigma_{\mathrm{th}}(T, \mu, \mu)$ where $M$ and $\sigma_{\text {th }}$ are given by Eqs. (31) and (11), respectively. The equation $M(\sigma)=\mu$ can be solved analytically ${ }^{8}$ :

$$
\sigma=\sigma(\mu)=\frac{a+\sqrt{a^{2}+4 b\left(\mu^{2}-m^{2}\right)}}{2 b} .
$$

As shown in Sec. II B, within the NRA one can use the approximate relations $\sigma_{\text {th }} \approx \frac{g \xi(3 / 2)}{\mu \lambda_{T}^{3}(T, \mu)}$ and $n \approx \mu \sigma(\mu)$. Finally we get Eq. (22) for the temperature $T_{\mathrm{BEC}}(\mu)$.

\section{B. Liquid-gas phase transition}

Let us consider first the states without BEC. At given $T, \mu$ one can solve Eqs. (10) and (31) with respect to $\sigma$. At sufficiently low temperatures there is a region of $\mu$ with several

\footnotetext{
${ }^{7}$ More exactly, one gets the 'stiff' $(\gamma=1)$ version of the potential introduced in Ref. [36].

${ }^{8}$ We have checked that the second root of this equation corresponds to unstable states.
} 
solutions $\sigma_{i}(\mu, T)$. The solution with the largest (lowest) pressure is thermodynamically stable (unstable) [43]. The critical line of the LGPT $\mu=\mu(T)$ is found from the Gibbs condition of phase equilibrium. The latter implies equality of pressure in coexisting liquidlike $(i=l)$ and gas-like $(i=g)$ domains of the mixed phase $(\mathrm{MP})^{9}$. Within our model one gets three coupled equations for $\mu(T), \sigma_{g}(T)$, and $\sigma_{l}(T)$ :

$$
\begin{aligned}
& p_{\text {th }}\left[T, \mu, M\left(\sigma_{g}\right)\right]+p_{\text {ex }}\left(\sigma_{g}\right)=p_{\text {th }}\left[T, \mu, M\left(\sigma_{l}\right)\right]+p_{\text {ex }}\left(\sigma_{l}\right), \\
& \sigma_{i}=\sigma_{\text {th }}\left[T, \mu, M\left(\sigma_{i}\right)\right] \quad(i=g, l) .
\end{aligned}
$$

Solving these equations gives the critical lines $\mu=\mu(T), p=p(T)$ of the LGPT and
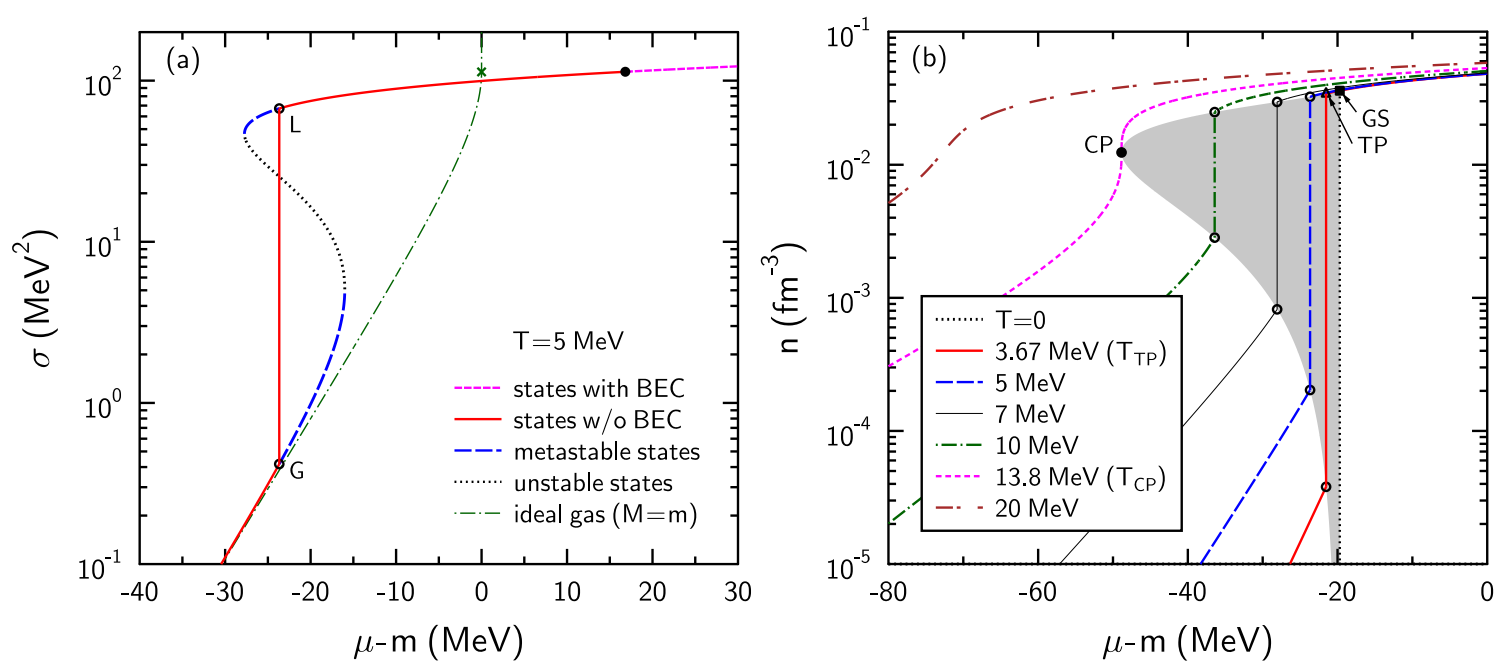

FIG. 2. (a) The isotherm $T=5 \mathrm{MeV}$ of $\alpha$ matter on the $(\mu, \sigma)$ plane. The solid and short-dashed lines show equilibrium states without and with BEC. The filled dot marks the boundary of the BEC states. The vertical section GL corresponds to MP states of the LGPT. The dashed and dotted lines represent the metastable and unstable states. The thin dashed-dotted curve corresponds to the ideal-gas limit. The cross shows the boundary of BEC states in the ideal gas. (b) Isotherms of $\alpha$ matter on the $(\mu, n)$ plane. The filled square, triangle and circle correspond, respectively, to the GS, TP and CP. The region of MP is shown by shading.

the values $\sigma_{i}(T), M_{i}(T), n_{i}(T), s_{i}(T) \ldots$ at the MP boundaries ('binodals') $i=g, l$. Note that within the MP region, $\mu$ and $p$ are functions of temperature only and do not depend, e.g., on density. The latter is connected with the volume fraction of the gas

${ }^{9}$ We neglect surface effects associated with finite sizes of domains. 
phase $\lambda \equiv V_{g} /\left(V_{g}+V_{l}\right) \in[0,1]:$

$$
n=\lambda n_{g}(T)+(1-\lambda) n_{l}(T) .
$$

At given $T, n$ one gets the relation

$$
\lambda=\frac{n_{l}(T)-n}{n_{l}(T)-n_{g}(T)} .
$$

Our calculations show that at $T=T_{\mathrm{TP}} \approx 3.67 \mathrm{MeV}$ (so-called triple point temperature) the BEC boundary reaches the $\mathrm{MP}$ region. At $T<T_{\mathrm{TP}}$, Bose condensate appears in the liquid-phase domains. In this case the conditions of phase equilibrium are obtained from Eqs. (39), (40) after replacing $M\left(\sigma_{l}\right)$ by $\mu$, and $\sigma_{l}$ by $\sigma(\mu)$ from Eq. (38).

A typical example for $T=5 \mathrm{MeV}$ is shown in Fig. 2 (a). The solid and short-dashed lines show equilibrium states on the $(\mu, \sigma)$ plane. At the considered temperature the BEC states lie outside the MP region (the vertical line GL). One can see that at large scalar densities the results strongly deviate from the ideal gas of bosons with the vacuum mass $m$. We also show positions of metastable and unstable (spinodal) states. They correspond to solutions of the gap equation with lower values of pressure as compared to equilibrium state at the same $T$ and $\mu$.

TABLE I. Characteristics of critical point of $\alpha$ matter

\begin{tabular}{|c|c|c|c|c|c|c|c|}
\hline$T(\mathrm{MeV})$ & $\mu-m(\mathrm{MeV})$ & $M-m(\mathrm{MeV})$ & $\sigma\left(\mathrm{MeV}^{2}\right)$ & $n\left(\mathrm{fm}^{-3}\right)$ & $S / N$ & $p /(n T)$ & $n \lambda_{T}^{3}$ \\
\hline 13.8 & -48.9 & -20.0 & 25.8 & 0.0124 & 4.54 & 0.334 & 0.129 \\
\hline
\end{tabular}

In Fig. 2 (b) we compare different isotherms of $\alpha$ matter on the $(\mu, n)$ plane. The lower and upper boundaries of the MP (shaded region) correspond, respectively to the gas-like and liquid-like binodals. Note that at $T=0$ the liquid-like binodal state coincides with the GS of equilibrium $\alpha$ matter. One can see that the LGPT disappears when $T$ exceeds the critical point $(\mathrm{CP})$ temperature $T_{\mathrm{CP}} \approx 13.8 \mathrm{MeV}$. At this point the derivative $(\partial n / \partial \mu)_{T}$ diverges. Table I shows characteristics of CP obtained within the present model.

Figure 3 represents the phase diagram on the $(\mu, T)$ plane. The MP region corresponds to the thick solid line between the GS and the CP. The BEC states lie below the thin curve which crosses the LGPT critical line at the triple point TP. The domain of BEC states is shown in Fig. 3 by the shaded area. At the same plot we show the behavior of adiabatic trajectories (isentropes), i.e., the lines of constant specific entropy $S / N=$ const. 


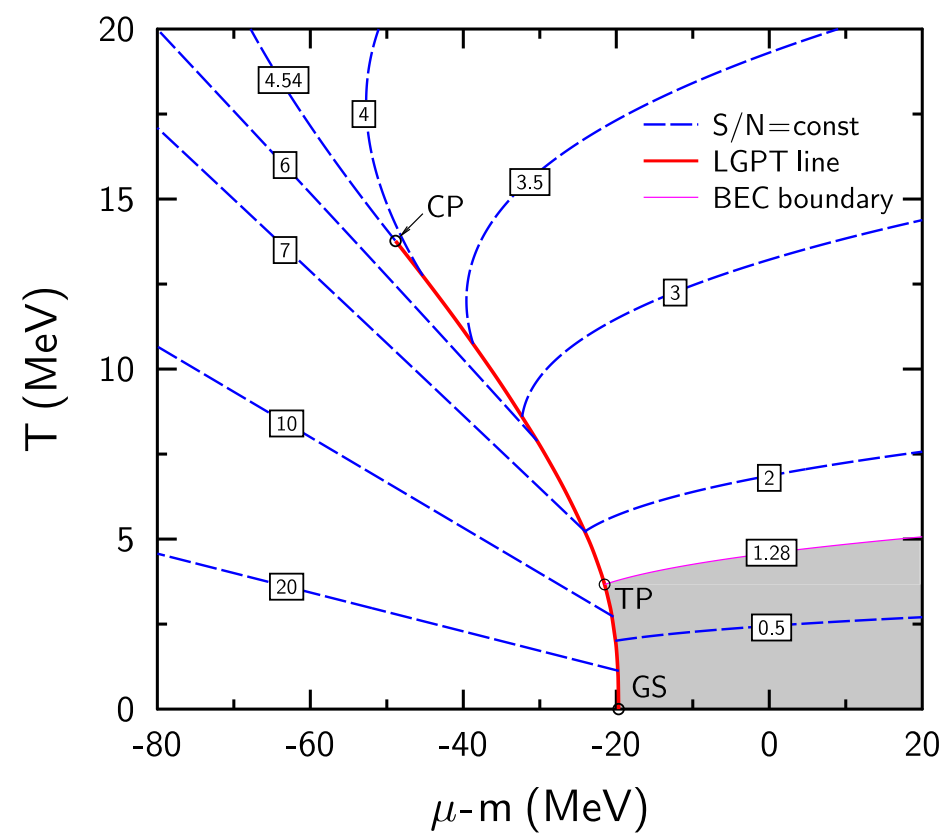

FIG. 3. Phase diagram of $\alpha$ matter on the $(\mu, T)$ plane. The dashed curves are adiabatic trajectories, i.e., lines of constant entropy per particle. The values of $S / N$ are given in boxes. The BEC region is shown by shading.

The isentropes play an important role in a fluid-dynamical evolution of excited matter ${ }^{10}$. In particular, we would like to mention the hydrodynamic [45] and thermal [46, 47] models of heavy-ion collisions which successfully describe particle production at high energies. They postulate that a hot and compressed 'fireball' is formed at some intermediate stage of a nuclear collision. Due to the presence of internal pressure, the fireball expands at later stages, producing secondary particles. Fitting the observed data confirmed that the specific entropy of fireball is fully determined by the initial c.m. energy of colliding nuclei.

As one can see in Fig. 3, the isentropes enter the MP region at any $S / N$. Especially important is the isentrope $S / N=(S / N)_{\mathrm{CP}} \approx 4.54$ which goes through the CP. As will be shown later, the trajectories with the specific entropy close to $(S / N)_{\mathrm{CP}}$ go through states with anomalously large fluctuations of the particle density. Note, that isentropes with $S / N$ larger (smaller) than $(S / N)_{\mathrm{CP}}$ enter the MP region at the gas-like (liquid-like) side. As mentioned above, the isentropes do not cross the BEC boundary outside the MP region. We shall come back to discussing this phase diagram in Sec. IV A (see Fig. 9).

${ }^{10}$ In absence of dissipation and shock waves, the total entropy is conserved for thermally equilibrated matter even in presence of collective flow. 


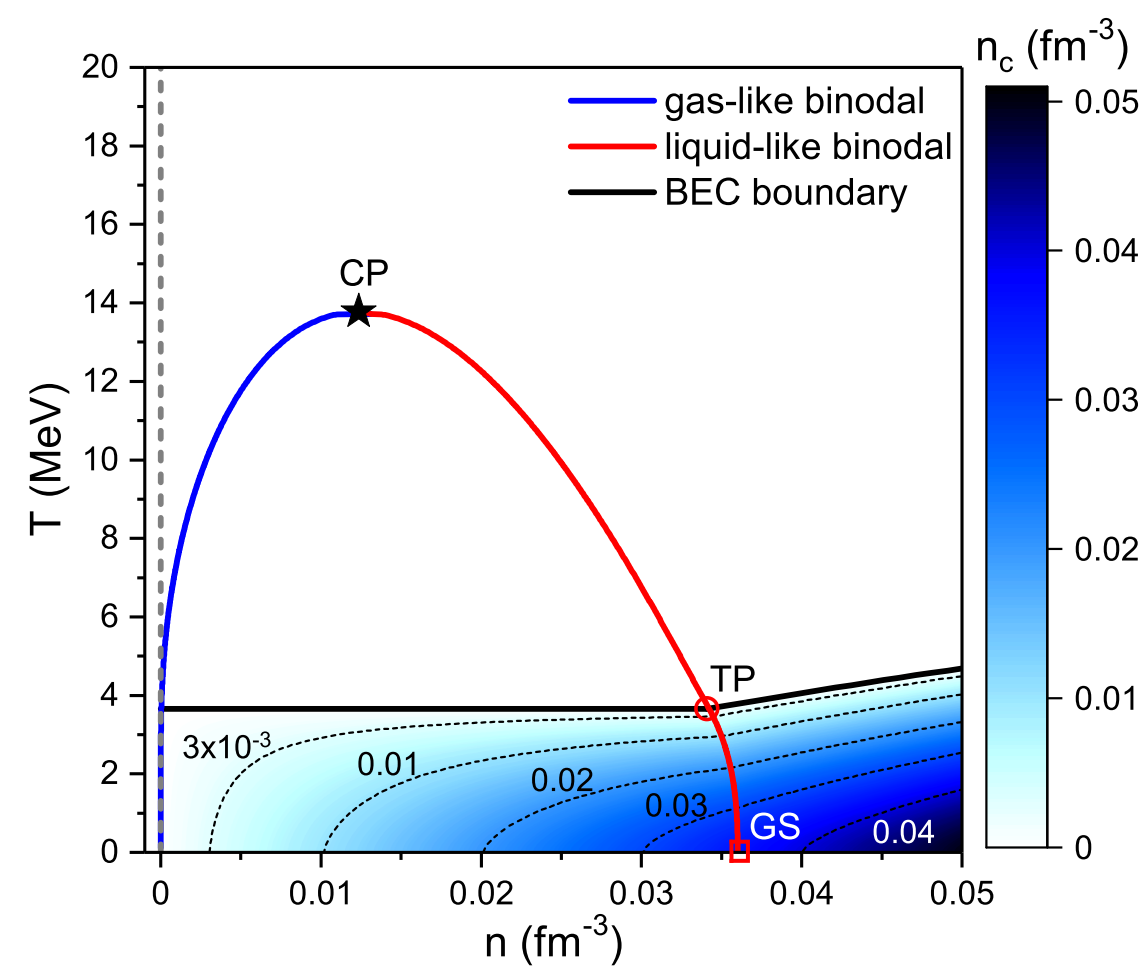

FIG. 4. Phase diagram of $\alpha$ matter on the $(n, T)$ plane. The values of the condensate density $n_{c}$ are shown by different shades of blue color (see the color map on r.h.s.). The dotted lines are contours of equal $n_{c}$ in the BEC region. The square, circle and star correspond, respectively, to the GS, TP, and CP.

Let us now calculate explicitly the condensate density $n_{c}$ at fixed $n, T$, first, for states outside the MP, i.e., for $n>n_{l}(T)$ and $T<T_{\mathrm{BEC}}$. As explained in Sec. II B this can be done by solving the equations

$$
n_{c}=\mu\left[\sigma(\mu)-\sigma_{\mathrm{th}}(T, \mu, \mu)\right]=n-n_{\mathrm{th}}(T, \mu, \mu),
$$

where $\sigma(\mu)$ is given by Eq. (38). One can see that $n_{c}$ increases from zero to $n$ when temperature drops from $T=T_{\mathrm{BEC}}$ to zero.

Inside the MP the condensate appears at $T<T_{\mathrm{TP}}$. At such temperatures the densities of the gas- and liquid-like domains satisfy the relation $n_{g}(T) \ll n_{l}(T)$. Unless the temperature is extremely low, one can disregard presence of BEC in the gaseous phase. Then one can write the relation $\left\langle n_{c}\right\rangle \approx(1-\lambda) n_{c l}(T)$ for the density of BEC averaged over the ensemble of coexisting domains. Here $n_{c l}(T)$ is the condensate density in the liquid phase at $T<T_{\mathrm{TP}}$. Substituting further Eq. (42) and neglecting terms $\sim n_{g} / n_{l}$ one gets the relation 

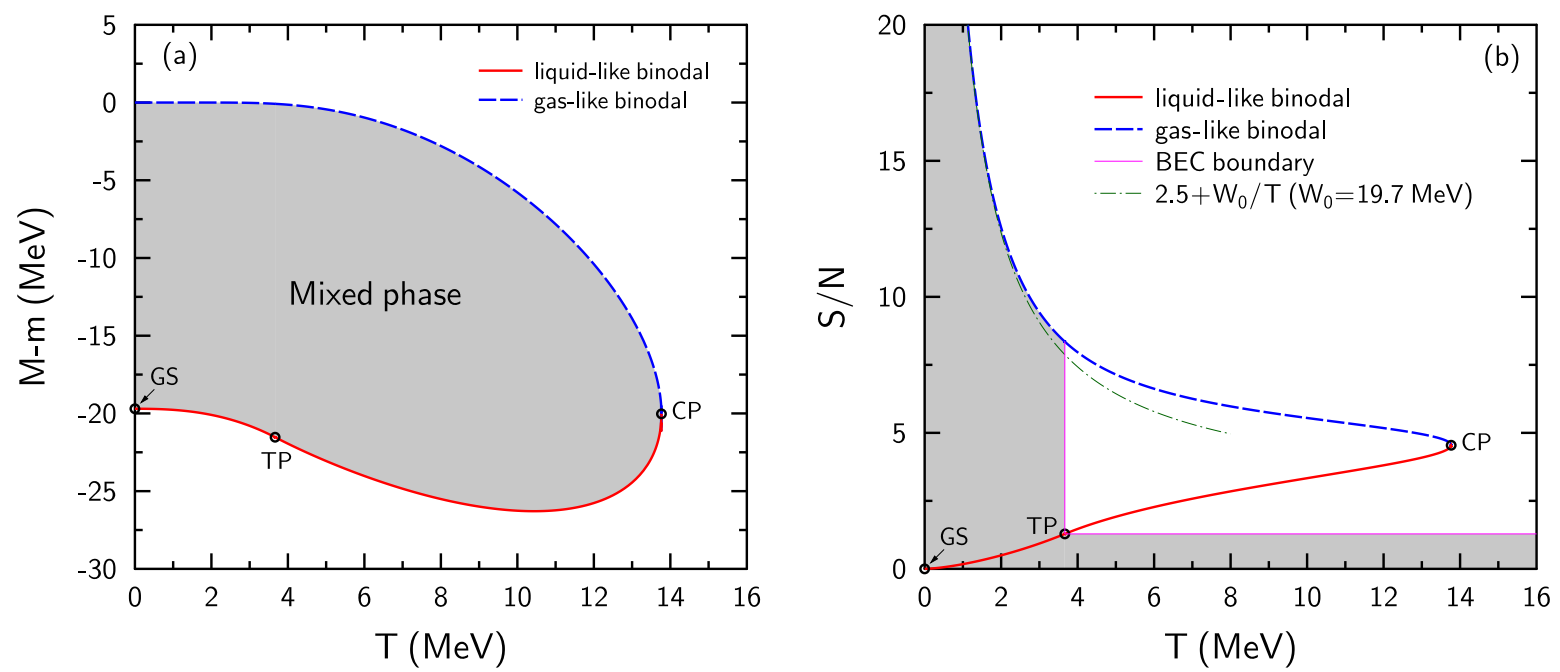

FIG. 5. Phase diagrams of $\alpha$ matter on the $(T, M)(\mathrm{a})$ and $(T, S / N)$ (b) planes. The regions of MP (a) and BEC (b) are shown by shading.

$\left\langle n_{c}\right\rangle / n \approx n_{c l}(T) / n_{l}(T)$. More detailed information is given in Fig. 4 where we show lines of equal condensate densities on the $(n, T)$ plane. The obtained phase diagrams are similar to those derived in Ref. [35]. It is interesting that they qualitatively agree with phase diagrams observed [48] for atomic ${ }^{4} \mathrm{He}$.

Figure 5 (a) shows the phase diagram on the $(T, M)$ plane. One can see that $M_{g}(T)>M_{l}(T)$. At low temperatures $M_{g}(T) \approx m$ and $M_{l}(T)=\mu(T) \approx m-W_{0}$ [see Eq. (34)]. In Fig. 5(b) we show the values of specific entropy $\widetilde{s}_{i}=(S / N)_{i}$ at the MP boundaries $i=g, l$. One can see that $\widetilde{s}_{l}(T)<\widetilde{s}_{\mathrm{CP}}<\widetilde{s}_{g}(T)$. It is possible to derive a simple analytic formula for $\widetilde{s}_{g}(T)$ at $T \rightarrow 0$. Indeed, in this limit the density $n=n_{g}(T)$ is small and one can apply the Boltzmann approximation in calculating the specific entropy on the gas-like binodal. One gets approximate relations

$$
\widetilde{s}_{g}(T) \approx \frac{5}{2}-\ln z \approx \frac{5}{2}+\frac{m-\mu(T)}{T} \approx \frac{5}{2}+\frac{W_{0}}{T}
$$

In the first equality we have used the upper line of Eq. (25). Comparison of the dashed and dash-dotted lines in Fig. 5(b) confirms good accuracy of these relations at low $T$.

In Fig. 6 we compare the behavior of isentropes $S / N=3$ and $S / N=5$ on the $(n, T)$ plane. Within the MP region we use the relation for the entropy density

$$
s=\lambda s_{g}(T)+(1-\lambda) s_{l}(T),
$$




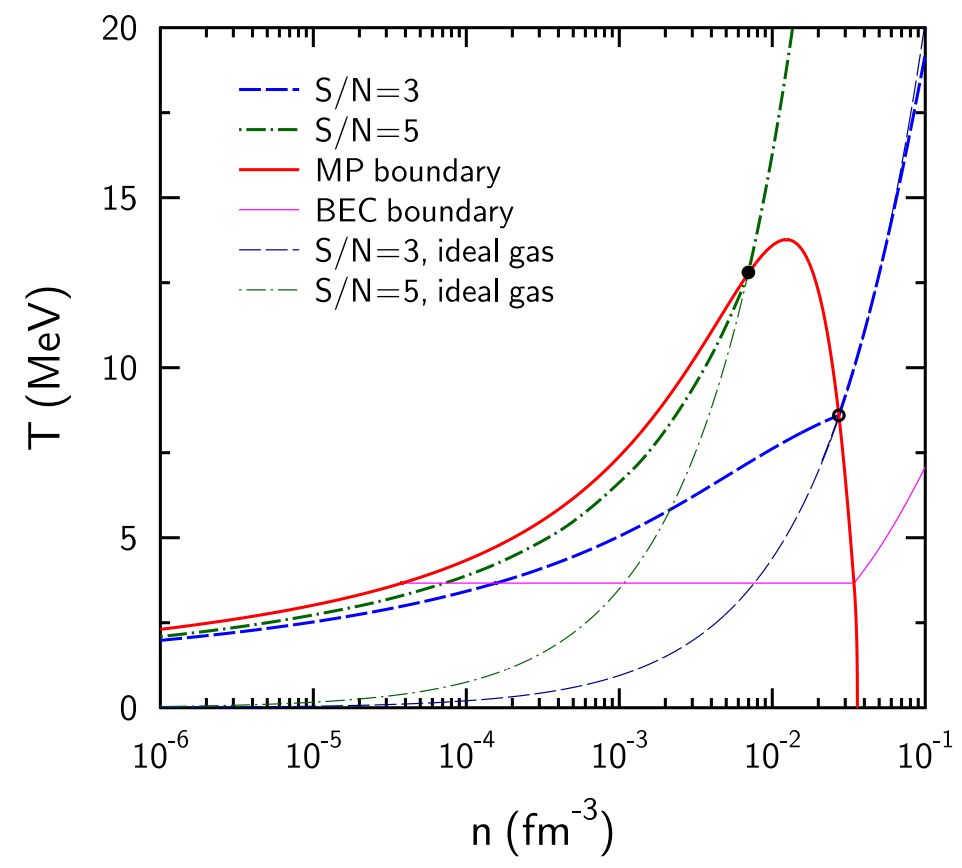

FIG. 6. The isentropes $S / N=3$ (the dashed line) and $S / N=5$ (the dash-dotted line) on the $(n, T)$ plane. The dots mark their intersection with the MP boundary. Thin dashed and dash-dotted lines represent the ideal-gas calculation.

where $\lambda$ is given by Eq. (42). One can see jumps in slopes of isentropes at the MP boundary (a similar behavior takes place also on the $(n, p)$ plane). This in turn leads to jumps of the sound velocity and the heat capacity at these boundaries (see Sec. IV B and IV C). Note that at small $n$ and $T$ isentropes go near the gas-like binodal. The calculation shows that outside the MP region the adiabatic trajectories are close to those in the ideal Bose-gas.

\section{POSSIBLE SIGNATURES OF PHASE TRANSITION IN $\alpha$ MATTER}

\section{A. Strong density fluctuations}

Statistical fluctuations of conserved charges (e.g., baryon number) are important observables for experimental studies of the phase diagrams of interacting systems. In the grand-canonical ensemble such fluctuations are expressed via 'susceptibilities', i.e., higherorder derivatives of pressure with respect to $T, \mu$. Below we calculate the scaled variance $\omega$ 
defined as the second moment of the particle number in a given volume:

$$
\omega=\frac{\left\langle N^{2}\right\rangle-\langle N\rangle^{2}}{\langle N\rangle},
$$

where averaging is performed at fixed temperature. Outside the MP region one gets [43] the relation

$$
\omega=\frac{T}{n}\left(\frac{\partial n}{\partial \mu}\right)_{T}=\frac{T}{n}\left(\frac{\partial^{2} p}{\partial \mu^{2}}\right)_{T} .
$$

In calculating the derivatives in the r.h.s. of (47) one should explicitly take into account the dependence $M=M(T, \mu)$.

At the region $T>T_{\mathrm{BEC}}$, using Eqs. (15), (31) and (10), one has

$$
\omega=\frac{T}{n}\left[\frac{\partial n_{\mathrm{th}}(T, \mu, M)}{\partial \mu}+\frac{\partial n_{\mathrm{th}}(T, \mu, M)}{\partial M} M^{\prime}(\sigma)\left(\frac{\partial \sigma}{\partial \mu}\right)_{T}\right],
$$

where

$$
M^{\prime}(\sigma)=\left(b \sigma-\frac{a}{2}\right) M^{-1}, \quad\left(\frac{\partial \sigma}{\partial \mu}\right)_{T}=\frac{\partial \sigma_{\mathrm{th}}}{\partial \mu}\left[1-\frac{\partial \sigma_{\mathrm{th}}}{\partial M} M^{\prime}(\sigma)\right]^{-1} .
$$

At $\mu \rightarrow \mu_{\mathrm{CP}}, T \rightarrow T_{\mathrm{CP}}$ both $(\partial n / \partial \mu)_{T}$ and $(\partial \sigma / \partial \mu)_{T}$ diverge which leads to the relation

$$
\frac{\partial \sigma_{\mathrm{th}}}{\partial M} M^{\prime}(\sigma)=1 \quad \text { at } \mathrm{CP} .
$$

As one can see from Eq. (11) the density $\sigma_{\text {th }}$ decreases with $M$ at fixed $T, \mu$. Therefore, the $\mathrm{CP}$ (and LGPT) may exist if $M(\sigma)$ contain regions with $M^{\prime}(\sigma)<0$. In accordance with first equality in Eq. (49), this is possible only if the interaction has an attractive term with nonzero $a$.

By using Eqs. (17) and (19) one can calculate the density derivatives entering Eq. (48) in the NRA. Then one obtains

$$
\left(\frac{\partial \sigma}{\partial \mu}\right)_{T} \approx \frac{\sigma g_{1 / 2}(z)}{\sigma M^{\prime}(\sigma) g_{1 / 2}(z)+T g_{3 / 2}(z)} .
$$

At given $n, T$, the values $z$ and $\sigma$ are determined from the approximate relations

$$
n \approx m \sigma \approx g g_{3 / 2}(z) / \lambda_{T}^{3}(T, m)
$$

Substituting (51) into (48) gives ${ }^{11}$

$$
\omega \approx\left[\frac{g_{3 / 2}(z)}{g_{1 / 2}(z)}+\frac{\sigma M^{\prime}(\sigma)}{T}\right]^{-1} .
$$

\footnotetext{
${ }^{11}$ A similar result can be obtained in the vector model of Ref. [35] with the replacement $\sigma M^{\prime}(\sigma) \rightarrow n U^{\prime}(n)$ where $U(n)$ is the potential introduced in Eq. (37).
} 
The second term in denominator vanishes in the limiting case of the ideal Bose gas $(M=m)$. Note, that at $T \rightarrow T_{\mathrm{BEC}}$ the first term in brackets goes to zero.

At $T<T_{\mathrm{BEC}}$ using Eq. (23) one has:

$$
\omega=\frac{T}{n}\left[\frac{\partial n_{\mathrm{th}}(T, \mu, \mu)}{\partial \mu}+\frac{\partial n_{c}(T, \mu)}{\partial \mu}\right]
$$

where, $n_{c}(T, \mu)$ is defined by the first equality in (43). Within the NRA one has approximately $n \approx \mu \sigma(\mu)$, where $\sigma(\mu)$ is given by Eq. (38). Using further Eq. (47) one gets the relations

$$
\omega \approx \frac{T \sigma^{\prime}(\mu)}{\sigma(\mu)}=\frac{\mu T}{\sigma(b \sigma-0.5 a)} .
$$

One can see that $\omega \rightarrow 0$ at $T \rightarrow 0$. This greatly deviates from the ideal-gas case $(a=0, b=0)$ where $\omega$ is infinite [43] in the BEC region.
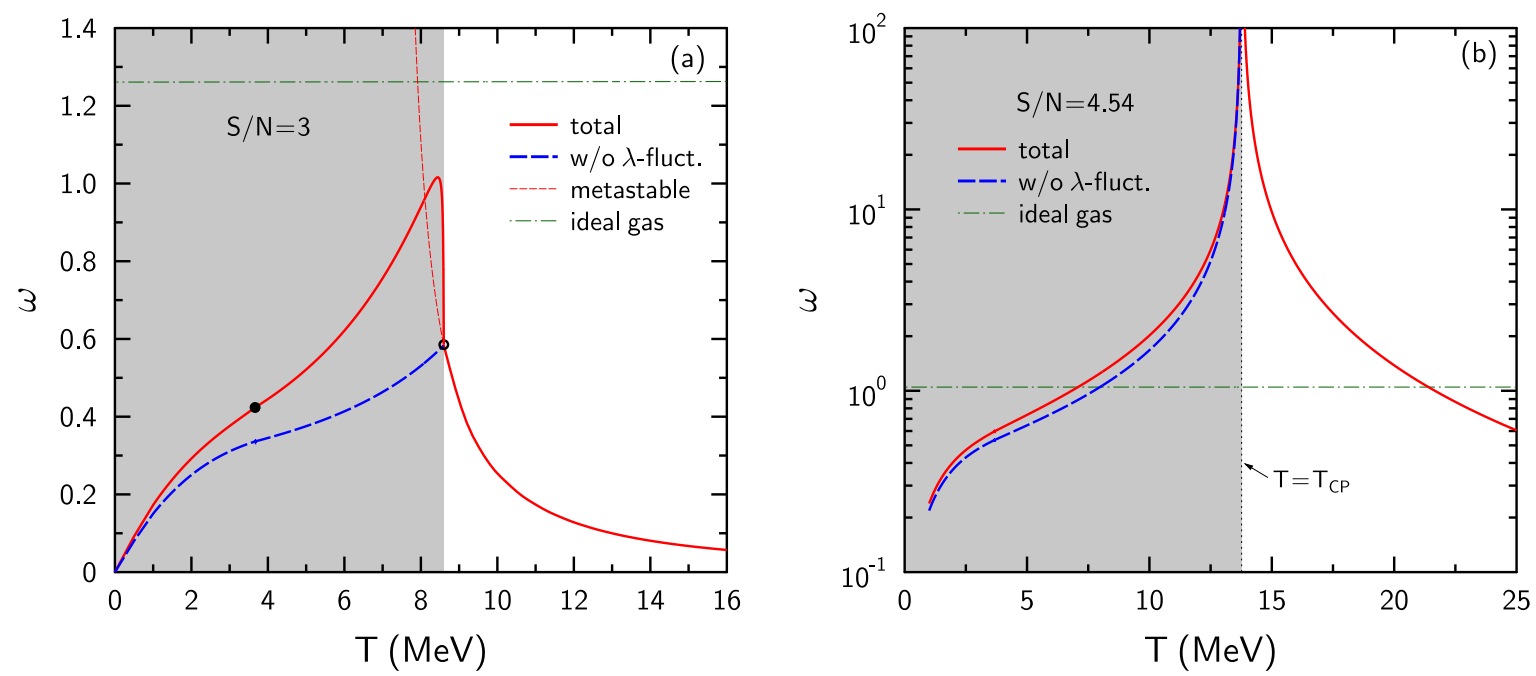

FIG. 7. Scaled variance of $\alpha$ matter as the function of temperature along the isentropes $S / N=3$ (a) and $S / N=4.54$ (b). The dashed lines are calculated without the third term in Eq. (56). The MP regions are shown by shading. The open and full dots correspond, respectively, to boundaries of MP and BEC states.

In the MP region one should take into account not only fluctuations of particle numbers inside the coexisting domains of matter, but also fluctuations of the interphase boundaries, which change the relative fraction of domain volumes. The latter corresponds to fluctuations of the parameter $\lambda$ around the equilibrium value given by Eq. (42). One can include both types of fluctuations by calculating $\omega$ directly from Eq. (46). In Ref. [49] such a calculation 


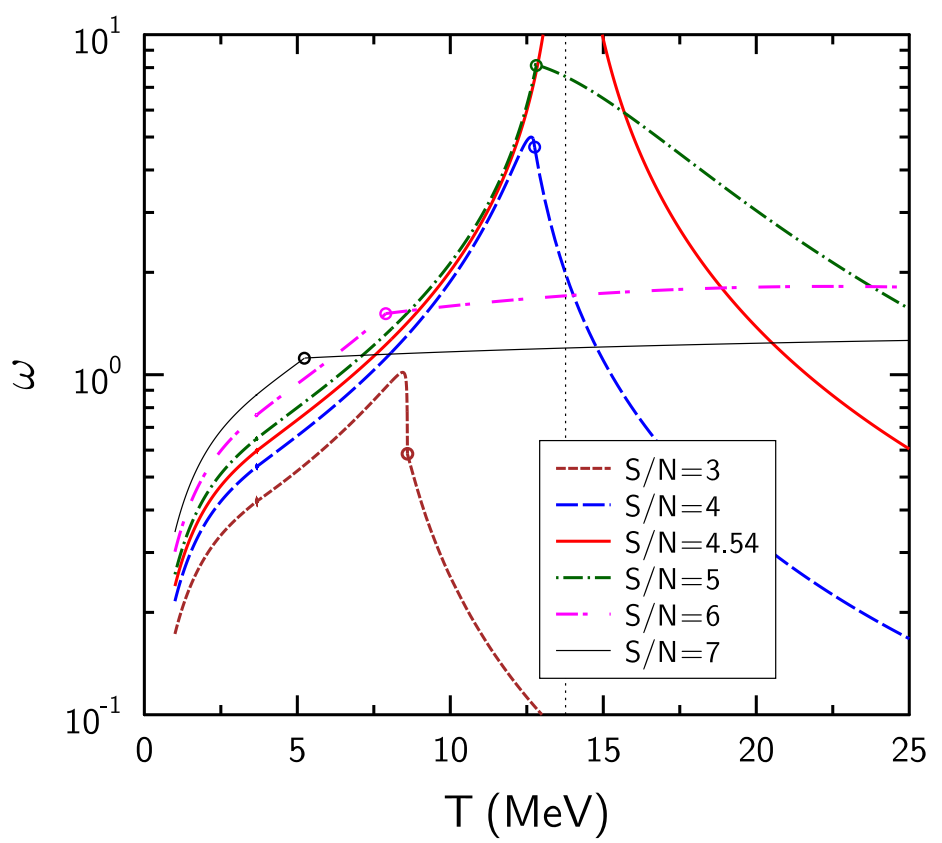

FIG. 8. Scaled variance of $\alpha$ matter as the function of temperature along the isentropes with $S / N=3-7$. Circles mark points where the isentropes cross the MP boundary. The vertical dotted line shows the temperature $T=T_{\mathrm{CP}}$.

was made for a particular case of the classical van der Waals model. Below we apply a similar approach for an arbitrary LGPT ${ }^{12}$.

We obtain the following result at given $n, T$ in the MP region:

$$
\omega=\frac{1}{n}\left[\lambda n_{g} \omega_{g}+(1-\lambda) n_{l} \omega_{l}+\left(n_{l}-n_{g}\right)^{2} \frac{\lambda(1-\lambda) \omega_{g} \omega_{l}}{\lambda n_{l} \omega_{g}+(1-\lambda) n_{g} \omega_{l}}\right] .
$$

Here $\lambda$ is defined in Eq. (42), $n_{i}$ and $\omega_{i}$ are, respectively, the particle densities and scaled variances inside the domains $i=g, l$. These quantities are equal to their values at the gasand liquid-like binodals. The contribution of $\lambda$ fluctuations is described by the third term in the r.h.s. of (56). This term vanishes near the MP boundary where $\lambda(1-\lambda) \rightarrow 0$.

The results of $\omega$ calculation for states along the isentropes with different $S / N$ are shown in Figs. 7-8. The calculations predict strong peaks of scaled variance for $S / N=4.5 \pm 1$. In Fig. 7 we make the comparison with the ideal-gas calculation. One can see the strong sensitivity of $\omega$ to particle interactions. Figures $9(\mathrm{a})$ and (b) show the density plots of $\omega$ in the $(\mu, T)$ and $(n, T)$ planes, respectively. One can see a very narrow peak of $\omega(T, \mu)$ at $T \approx T_{\mathrm{CP}}$. This can be used as a clear signal of the CP in experimental searches of LGPT.

${ }^{12}$ Higher-order particle number fluctuations can be calculated by using the method developed recently in Ref. [50]. 

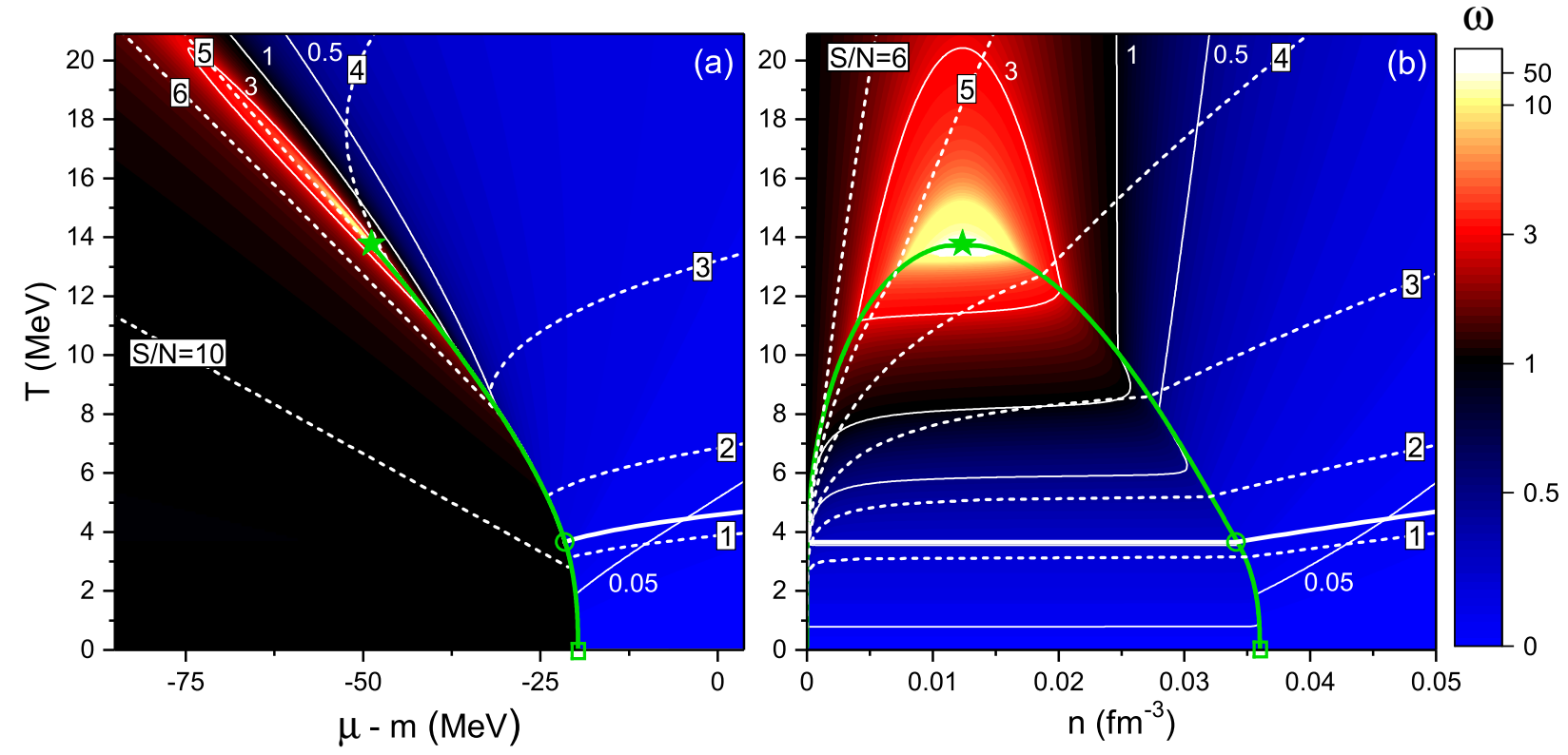

FIG. 9. (a) The dashed lines: isentropic trajectories with different $S / N$ (shown by black numbers in boxes) on the $(\mu, T)$ plane. Colors show values of scaled variance $\omega$. Thin white lines are contours of equal $\omega$ (their values are given by white numbers). The thick green and white lines represent the LGPT line and the BEC boundary, respectively. The star marks position of the CP. (b) Same as (a) but on the $(n, T)$ plane. Note the strong $\omega$ peak near the critical point.

\section{B. Softening of the equation of state}

An important characteristics of the equation of state is the sound velocity $c_{s}$ which characterizes propagation of a small perturbation in the local rest frame of matter. In the ideal fluid dynamics the sound velocity squared is equal to [43]

$$
c_{s}^{2}=\left(\frac{\partial p}{\partial \varepsilon}\right)_{\widetilde{s}}=\left(\frac{\partial p}{\partial \varepsilon}\right)_{n}+\frac{n}{\varepsilon+p}\left(\frac{\partial p}{\partial n}\right)_{\varepsilon},
$$

where $\widetilde{s}=s / n$ is the entropy per particle ${ }^{13}$. Using Eqs. (27), (28) one can directly calculate $c_{s}$ by expressing the derivatives entering Eq. (57) via the derivatives of $n, s$ over $T, \mu$. For the MP states one can use Eqs. (41), (45) and rewrite these derivatives via the binodal quantities $s_{i}^{\prime}(T)$ and $n_{i}^{\prime}(T)(i=g, l)$.

${ }^{13}$ Equation (57) is derived for a continuous matter without large gradients of density. On the other hand, this is not true for MP with different densities of liquid and gas domains. Nevertheless, one can approximately consider the MP region as a homogeneous matter if the wavelength of a sound wave exceeds typical domain sizes (i.e., at low enough frequencies). 
Within the NRA one can get much simpler expressions (outside the MP). Indeed, from the first equality in (57), substituting $\varepsilon \approx m n$ one has

$$
c_{s}^{2} \approx \frac{1}{m}\left[\left(\frac{\partial p_{\mathrm{th}}}{\partial n}\right)_{\widetilde{s}}+\left(\frac{\partial p_{\mathrm{ex}}}{\partial n}\right)_{\widetilde{s}}\right]
$$

According to Eq. (25), in this approximation the specific entropy $\widetilde{s}$ depends in the NRA only on fugacity $z$ at $T>T_{\mathrm{BEC}}$ or on $n \lambda_{T}^{3}$ at $T<T_{\mathrm{BEC}}$. From Eqs. (17) and (18) one can see that in both cases $n T^{-2 / 3}$ and $p_{\text {th }} T^{-5 / 3}$ are approximately constant at $\widetilde{s}=$ const. Using Eq. (6) one gets

$$
c_{s}^{2} \approx \frac{\sigma M^{\prime}(\sigma)}{m}+\frac{5 T}{3 m} \begin{cases}\frac{g_{5 / 2}(z)}{g_{3 / 2}(z)}, & T>T_{\mathrm{BEC}}, \\ \frac{\xi(5 / 2)}{n \lambda_{T}^{3}(T, m)}, & T<T_{\mathrm{BEC}}\end{cases}
$$

where $\sigma \approx n / m$ and $z$ is found by solving the equation $n \lambda_{T}^{3}(T, m) / g=g_{3 / 2}(z)$. The second term in $(59)$ is the same as for ideal Bose-gas ${ }^{14}$, and the first one takes into account the interaction effects.
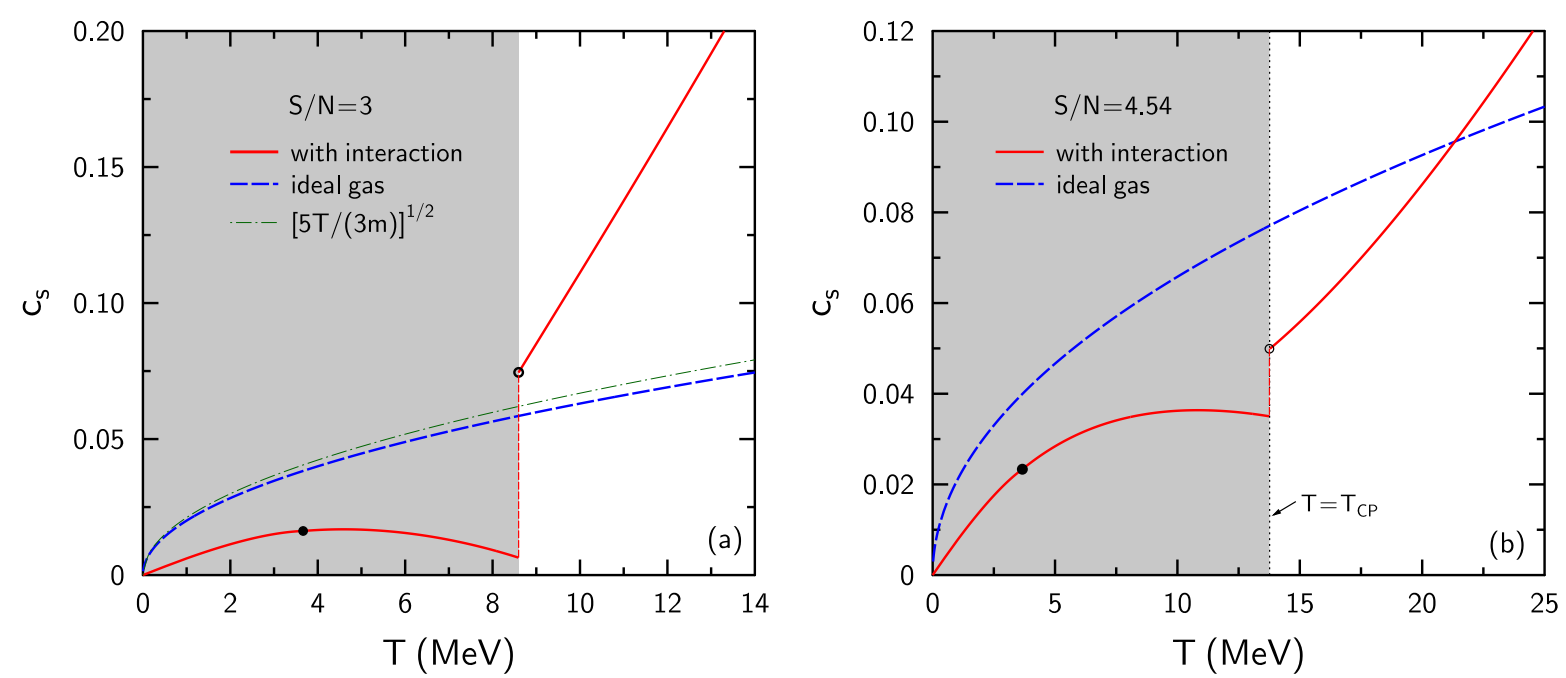

FIG. 10. Sound velocity as the function of temperature along the isentropes $S / N=3$ (a) and $S / N=4.54$ (b) (the solid lines). The dashed curves correspond to ideal gas. The MP states are shown by shading. Full dots mark the BEC boundary at $T=T_{\mathrm{TP}}$.

The results of calculation are presented in Figs. 10 (a) and (b) for states along the isentropes $S / N=3$ and $S / N=4.54$, respectively. As expected, the sound velocity is strongly

\footnotetext{
${ }^{14}$ In the Boltzmann approximation $c_{s}=\sqrt{5 T /(3 m)}$.
} 
suppressed inside the MP region. On the other hand, it becomes much larger at higher densities where the repulsive interaction dominates. Outside the MP region $c_{s}$ increases with $T$ faster as compared to the ideal-gas calculation. One can see discontinuities of sound velocities at the MP boundary: $c_{s}$ jumps down during the adiabatic expansion. It is known [51] that such a behavior leads to the formation of a rarefaction shock. The latter may be regarded as a signature of the LGPT.

\section{Enhanced heat capacity}

The isochoric heat capacity is another observable which is sensitive to the LGPT and BEC effects. This quantity (per particle) is defined as [43]

$$
c_{v}=\frac{T}{n}\left(\frac{\partial s}{\partial T}\right)_{n}=\frac{1}{n}\left(\frac{\partial \varepsilon}{\partial T}\right)_{n} .
$$

We calculate $c_{v}$ outside the MP by using formulae similar to Eqs. (48), (49). For states within the MP we perform the direct calculation based on Eqs. (41) and (45).

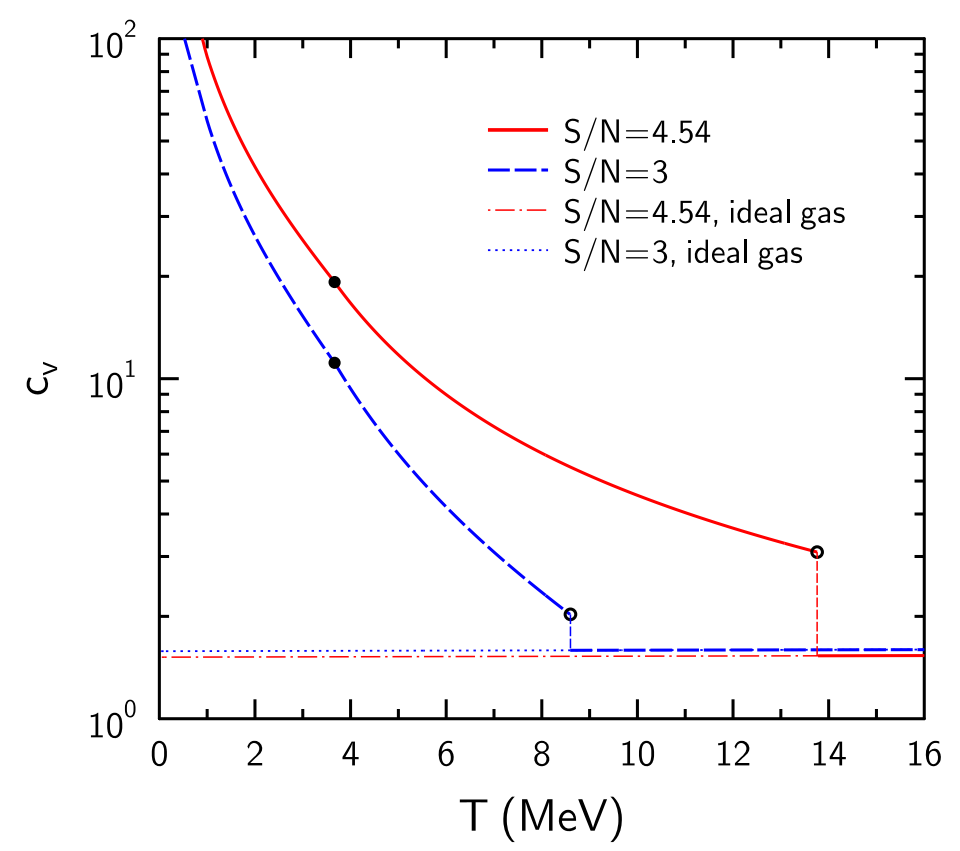

FIG. 11. Isochoric heat capacity per particle as the function of temperature along the isentropes $S / N=3$ (dashed) and $S / N=4.54$ (solid). Full dots correspond to $T=T_{\mathrm{TP}}$. Open dots mark the MP boundary. Thin dotted and dash-dotted lines represent the ideal gas calculation.

Within the NRA one can use approximate relations (17), (25) with $M \approx m$. Outside 
the MP, we obtain the result coinciding with that for the ideal Bose gas [42]

$$
c_{v} \approx \begin{cases}\frac{15}{4} \frac{g_{5 / 2}(z)}{g_{3 / 2}(z)}-\frac{9}{4} \frac{g_{3 / 2}(z)}{g_{1 / 2}(z)}, & T>T_{\mathrm{BEC}} \\ \frac{15}{4} \frac{\xi(5 / 2)}{n \lambda_{T}^{3}(T, m)}, & T<T_{\mathrm{BEC}} .\end{cases}
$$

In the Boltzmann approximation one gets the well-known value $c_{v}=3 / 2$. At the BEC boundary $c_{v}=3.75 \xi(5 / 2) / \xi(3 / 2) \approx 1.925$.

Figure 11 shows the temperature dependence of $c_{v}$ along the isentropes with same $S / N$ as in Fig. 10. Again one can see jumps of the heat capacity at the MP boundary. The predicted $c_{v}$-values are much larger in the MP region as compared to the ideal gas calculation. These values increase roughly as $T^{-1}$ at $T \rightarrow 0$. Such a behavior can be qualitatively understood. Indeed, as mentioned above, at low temperatures the isentrope $\widetilde{s}=$ const becomes close to the gas-like binodal, i.e., $\lambda \approx 1$ and $n \approx n_{g}(T)$. Substituting $s \approx \widetilde{s} n_{g}(T)$ into Eq. (60) gives

$$
\frac{c_{v}}{\widetilde{s}} \approx T \frac{n_{g}^{\prime}(T)}{n_{g}(T)} \approx \frac{m-\mu(T)}{T} \approx \frac{W_{0}}{T} .
$$

In the second equality we neglect deviations from the Boltzmann statistics having in mind that the density $n_{g}$ is small at low temperatures.

\section{CONCLUSIONS}

We have proposed a field-theoretical model to describe $\alpha$-clustered nuclear matter at finite temperatures. The system of interacting $\alpha$ particles is represented by a scalar field $\phi$ with the Lagrangian containing the attractive $\left(\phi^{4}\right)$ and repulsive $\left(\phi^{6}\right)$ self-interaction terms. The calculations are done within the mean-field approach which obeys a self-consistency relation between the scalar mean field and the particle effective mass. The model has two free parameters which are fixed by fitting properties of the ground state of cold $\alpha$ matter known from microscopic calculations of Clark and Wang [15].

Our main results are as follows: 1) $\alpha$ matter exhibits a liquid-gas phase transition with the critical point at $T_{c} \approx 14 \mathrm{MeV}, n_{c} \approx 0.012 \mathrm{fm}^{-3} ; 2$ ) at low temperatures the $\alpha$ matter contains the Bose-Einstein condensate, which appears in the liquid phase; 3) all isentropic trajectories, $S / N=$ const, terminate in the mixed-phase region; 4) the BEC boundary outside the mixed phase coincides with the isentrope $S / N \approx 1.28$; 5) the scaled variance 
of density fluctuations has a strong peak at the critical point which lies on the isentrope $S / N \approx 4.5 ; 6)$ the sound velocity and the isochoric heat capacity exhibit jumps at the mixed phase boundary.

Based on these results, we can formulate a strategy for experimental studies of $\alpha$-clustered matter. We believe that enhanced yields of $\alpha$ particles and $\alpha$-conjugate nuclei observed in Refs. $[2,10]$ are associated with the mixed phase of $\alpha$ matter formed at a late stage of the nuclear matter evolution. Since at this stage the expansion is approximately isentropic, one may hope to select events which correspond to the critical point of $\alpha$ matter around $S / N \approx 4.5$. In the vicinity of this point we expect a wide (power-law) mass distribution of produced fragments, like $Y(A) \propto A^{-\tau}$ where $\tau \approx 2$ is the Fischer exponent [7] .

Interesting manifestations of $\alpha$ clustering can be expected in heavy and superheavy nuclei. As demonstrated in Ref. [52], due to strong Coulomb repulsion such nuclei may develop a hollow structure where $\alpha$ 's are condensed in the outer shell but neutrons fill the central region. We hope that future experiments with heavy-ion beams at intermediate energies will provide new evidence for $\alpha$ clustering and $\alpha$ condensation in nuclear systems.

\section{ACKNOWLEDGMENTS}

The authors thank D. Anchishkin, M. Gorenstein, and V. Vovchenko for fruitful discussions. L.M.S, R.V.P. and I.N.M. thank the support from the Frankfurt Institute for Advanced Studies. H.St. appreciates the support from J. M. Eisenberg Laureatus chair and the W. Greiner Gesellschaft.

[1] A. Schüttauf et al., Nucl. Phys. A 607, 457 (1996).

[2] W. Reisdorf et al. (FOPI Collaboration), Nucl. Phys. A 848, 366 (2010).

[3] R. Wada, W. Lin, P. Ren, H. Zheng, X. Liu, M. Huang, K. Yang, and K. Hagel, Phys. Rev. C 99, 024616 (2019).

[4] D. E. H. Gross, Phys. Scripta T5, 213 (1983).

[5] J. P. Bondorf, I. N. Mishustin, C. J. Pethick, H. Schultz, and K. Sneppen, Nucl. Phys. A 443, 321 (1985).

[6] G. Peilert, J. Randrup, H. Stoecker and W. Greiner, Phys. Lett. B 260, 271 (1991). 
[7] J. P. Bondorf, A. S. Botvina, A. S. Ilinov, I. N. Mishustin, and K. Sneppen, Phys. Rept. 257, 133 (1995).

[8] P. Marini et al. (INDRA Collaboration), Phys. Lett. B 756, 194 (2016).

[9] B. Borderie et al. Phys. Lett. B 755, 475 (2016).

[10] K. Schmidt et al., Phys. Rev. C 95, 054618 (2017).

[11] D. A. Artemenkov et al., Eur. Phys. J. A 56, 250 (2020).

[12] M. Barbui et al. (FOPI Collaboration), Phys. Rev. C 98, 044601 (2018).

[13] P. Schuck, Y. Funaki, H. Horiuchi, G. Röpke, A. Tonsaki, and T. Yamada, Phys. Scripta 91, 123001 (2016).

[14] X. G. Cao et al., JPS Conf. Proc. 32, 010038 (2020).

[15] J. W. Clark and T.-P. Wang, Ann. Phys. 40, 127 (1966).

[16] M. T. Johson and J. W. Clark, Kinam 2, 3 (1980).

[17] C. J. Horowitz and A. Schwenk, Nucl. Phys. A 776, 55 (2006).

[18] X.-H. Wu, S.-B. Wang, A. Sedrakian, and G. Röpke, J. Low Temp. Phys. 189, 133 (2017).

[19] A. Sedrakian, Eur. Phys. J. A 56, 258 (2020).

[20] M. Freer, H. Horiuchi, Y. Kanada-En'yo, D. Lee, and U.-G- Meißner, Rev. Mod. Phys. 90, 035004 (2018).

[21] H. Horiuchi, Nucl. Phys. A 522, 257c (1991).

[22] H. Feldmeier, Nucl. Phys. A 515, 147 (1990).

[23] S. C. Pieper, R. B. Waringa, and V. R. Pandharipande, Phys. Rev. C 46, 1741 (1992).

[24] E. Epelbaum, H. Krebs, D. Lee, and U.-G- Meißner, Phys. Rev. Lett. 106, 192501 (2011).

[25] S. Elhatisari et al., Phys. Rev. Lett. 117, 132501 (2016).

[26] J.-P. Ebran, E. Khan, T. Niksic, and D. Wretenar, Nature 487, 341 (2012).

[27] J.-P. Ebran, E. Khan, T. Niksic, and D. Wretenar, Phys. Rev. C 90, 054329 (2014).

[28] J.-P. Ebran, M. Girod, E. Khan, R. D. Losseri, and P. Schuck, Phys. Rev. C 102, 014305 (2020).

[29] S. Misicu, I. N. Mishustin, and W. Greiner, Mod. Phys. Lett. A 32, 1750010 (2016).

[30] H. Pais, F. Gulminelli, C. Providéncia, and G. Röpke, Phys. Rev. C 97, 045805 (2018).

[31] Z.-W. Zhang and L. W. Chen, Phys. Rev. C 100, 054304 (2019).

[32] S. Furusawa and I. N. Mishustin, Nucl. Phys. A 1002, 121991 (2020).

[33] G. Peilert, J. Konopka, H. Stöcker, W. Greiner. M. Blann, and M. G. Mustafa, Phys. Rev. 
C 46, 1457 (1992).

[34] T. Maryama et al., Phys. Rev. C 57, 655 (1998).

[35] L. M. Satarov, M. I. Gorenstein, A. Motornenko, V. Vovchenko, I. N. Mishustin, and H. Stoecker, J. Phys. G: Nucl. Part. Phys. 44, 125102 (2017).

[36] L. M. Satarov, I. N. Mishustin, A. Motornenko, V. Vovchenko, M. I. Gorenstein, and H. Stoecker, Phys. Rev. C 99, 024909 (2019).

[37] L. M. Satarov, M. I. Gorenstein, I. N. Mishustin, and H. Stoecker, Phys. Rev. C 101, $024913(2020)$.

[38] J. Boguta and A. R. Bodmer, Nucl. Phys. A 292, 413 (1977).

[39] J. Boguta and H. Stoecker, Phys. Lett. B 120, 289 (1983).

[40] I. N. Mishustin, D. V. Anchishkin, L. M. Satarov, O. S. Stashko, and H. Stoecker, Phys. Rev. C 100, 022201 (2019).

[41] G. Baym, J.-P. Blaizot, M. Holzmann, F. Laloë, and D. Vautherin, Eur. Phys. J. B 24, 107 (2001).

[42] R. K. Pathria and P. D. Beale, Statistical Mechanics, (Elsevier, Amsterdam, 2011).

[43] L. D. Landau and E. M. Lifshitz, Statistical Physics (Pergamon, Oxford, 1975).

[44] O. Savchuk, Y. Bondar, O. Stashko, R. V. Poberezhnyuk, V. Vovchenko, M. I. Gorenstein, and H. Stoecker, Phys. Rev. C 102, 035202 (2020).

[45] L. D. Landau, Izv. Akad. Nauk SSSR, Ser. Fiz. 17, 51 (1953); in Collected Papers of L. D. Landau (Gordon and Breach, New York, 1965), p. 569.

[46] J. Cleymans and K. Redlich, Phys. Rev. Lett. 81, 5284 (1998).

[47] A. Andronic, P. Braun-Munzinger, and J. Stachel, Nucl. Phys. A 772, 167 (2006).

[48] H. Buchenau, E. L. Knuth, J. Northby, J. P. Toennies, and C. Winkler, J. Chem. Phys. 92, $6875(1990)$.

[49] V. Vovchenko, D. V. Anchishkin, M. I. Gorenstein, J. Phys. A: Math. Theor. 48, 305001 (2015).

[50] R. V. Poberezhnyuk, O. Savchuk, M. I. Gorenstein, V. Vovchenko, and H. Stoecker, arXiv: 2011.06420 [hep-ph].

[51] Ya. B. Zel'dovich and Yu. P. Reizer, Physics of Shock Waves and High-Temperature Hydrodynamic Phenomena (Academic Press, New York, 1966/1967).

[52] S. Misicu, I. N. Mishustin, in Walter Greiner Memorial Volume, eds. O. Hess, H. Stöcker, 
(World Scientific, Singapore, 2018), p. 263; arXiv: 1806.01886 [nucl-th]. 